\title{
Clinical Characteristics and Prognostic Factors for Intensive Care Unit Admission of Patients With COVID-19: Retrospective Study Using Machine Learning and Natural Language Processing
}

Jose Luis Izquierdo $^{1}, \mathrm{MD}$; Julio Ancochea ${ }^{2}$, MD; Savana COVID-19 Research Group ${ }^{3}$; Joan B Soriano ${ }^{2}$, MD, PhD

${ }^{1}$ Hospital Universitario de Guadalajara, Guadalajara, Spain

${ }^{2}$ Hospital Universitario de La Princesa, Madrid, Spain

${ }^{3}$ Savana, Madrid, Spain

Corresponding Author:

Joan B Soriano, MD, PhD

Hospital Universitario de La Princesa

Diego de León 62

Madrid, 28005

Spain

Phone: 34618867769

Email: jbsoriano2@gmail.com

\section{Related Articles:}

Comment in: https://www.jmir.org/2021/5/e27142

Comment in: https://www.jmir.org/2021/5/e29405

\section{Abstract}

Background: Many factors involved in the onset and clinical course of the ongoing COVID-19 pandemic are still unknown. Although big data analytics and artificial intelligence are widely used in the realms of health and medicine, researchers are only beginning to use these tools to explore the clinical characteristics and predictive factors of patients with COVID-19.

Objective: Our primary objectives are to describe the clinical characteristics and determine the factors that predict intensive care unit (ICU) admission of patients with COVID-19. Determining these factors using a well-defined population can increase our understanding of the real-world epidemiology of the disease.

Methods: We used a combination of classic epidemiological methods, natural language processing (NLP), and machine learning (for predictive modeling) to analyze the electronic health records (EHRs) of patients with COVID-19. We explored the unstructured free text in the EHRs within the Servicio de Salud de Castilla-La Mancha (SESCAM) Health Care Network (Castilla-La Mancha, Spain) from the entire population with available EHRs (1,364,924 patients) from January 1 to March 29, 2020. We extracted related clinical information regarding diagnosis, progression, and outcome for all COVID-19 cases.

Results: A total of 10,504 patients with a clinical or polymerase chain reaction-confirmed diagnosis of COVID-19 were identified; 5519 (52.5\%) were male, with a mean age of 58.2 years (SD 19.7). Upon admission, the most common symptoms were cough, fever, and dyspnea; however, all three symptoms occurred in fewer than half of the cases. Overall, 6.1\% (83/1353) of hospitalized patients required ICU admission. Using a machine-learning, data-driven algorithm, we identified that a combination of age, fever, and tachypnea was the most parsimonious predictor of ICU admission; patients younger than 56 years, without tachypnea, and temperature $<39$ degrees Celsius (or $>39^{\circ} \mathrm{C}$ without respiratory crackles) were not admitted to the ICU. In contrast, patients with COVID-19 aged 40 to 79 years were likely to be admitted to the ICU if they had tachypnea and delayed their visit to the emergency department after being seen in primary care.

Conclusions: Our results show that a combination of easily obtainable clinical variables (age, fever, and tachypnea with or without respiratory crackles) predicts whether patients with COVID-19 will require ICU admission.

(J Med Internet Res 2020;22(10):e21801) doi: 10.2196/21801

\section{KEYWORDS}

artificial intelligence; big data; COVID-19; electronic health records; tachypnea; SARS-CoV-2; predictive model 


\section{Introduction}

The unprecedented global spread of SARS-CoV-2, the virus that causes COVID-19, requires innovative approaches that deliver real-time results [1,2]. To date, big data analytics have been primarily used to assess SARS-CoV-2 transmission [3] and to indirectly estimate COVID-19 incidence using data from social media [4]. However, many factors involved in the onset and temporal distribution of the ongoing COVID-19 pandemic remain unknown. Similarly, both the individual and population burdens of COVID-19 are only beginning to be elucidated. Although big data analytics and artificial intelligence (AI) are widely used in the realms of health and medicine [5-7], researchers are only beginning to use these tools to explore the clinical characteristics and predictive factors of patients with COVID-19, including mortality [8-11].

Considering the unprecedented spread and severity of the ongoing COVID-19 outbreak, focus has been given to hospitals' unmet needs, particularly their ICU requirements $[8,9,12]$. Indeed, health systems have been or currently are near collapse, and independent modelling efforts have aimed at forecasting a number of epidemiological estimators, including ICU use [13-15].

Previously, our team reported that a combination of big data analytics and machine learning techniques helped better determine the quality of diagnosis and treatment of chronic obstructive pulmonary disease (COPD) via an analysis of hospital electronic health records (EHRs) using natural language processing (NLP) and validated algorithms [16,17].

As part of the BigCOVIData study, our primary objectives are to describe the clinical characteristics and determine the factors that predict ICU admission of patients with COVID-19. Determining these factors using a well-defined population can increase our understanding of the real-world epidemiology of the disease. To achieve this aim, we used a combination of classic epidemiological methods [18], NLP, and machine learning (for predictive modeling) to analyze the clinical information contained in the EHRs of patients with COVID-19.

\section{Methods}

The BigCOVIData study was conducted in compliance with legal and regulatory requirements and followed generally accepted research practices described in the International Council for Harmonisation of Technical Requirements for Pharmaceuticals for Human Use (ICH) Guideline for Good Clinical Practice, the latest edition of the Helsinki Declaration, the Guidelines for Good Pharmacoepidemiology Practices, and applicable local regulations. This study was classified as a "non-postauthorization study" by the Spanish Agency of Medicines and Health Products, and it was approved by the Research Ethics Committee at the University Hospital of Guadalajara (Spain). We and endorsed the STrengthening the Reporting of OBservational studies in Epidemiology (STROBE) guidance for reporting observational research [19].

\section{Study Design and Data Source}

This was a multicenter, noninterventional, retrospective study using data captured in the EHRs of the participating hospitals within the Servicio de Salud de Castilla-La Mancha (SESCAM) Health Care Network in Castilla-La Mancha, Spain (Figure 1). Data captured in EHRs were collected from all available departments, including inpatient hospital, outpatient hospital, and emergency department (ED), for virtually all types of provided services in each participating hospital. The study period was January 1 to March 29, 2020.

The study database was fully anonymized in a structured format and contained no personal information from patients. Likewise, personal information was not accessed during either the application of automated and algorithmic methods (ie, NLP) or the conversion of unstructured data into the structured database. Importantly, given that clinical information was handled in an aggregate, anonymized, and irreversibly dissociated manner, patient consent regulations do not apply to the present study. 
Figure 1. Map of the Castilla-La Mancha region (red) within the Spanish (blue line) and European territories. From a general source population of 2,035,000 inhabitants, we collected and analyzed the clinical information in the EHRs of 1,364,924 patients within the Servicio de Salud de Castilla-La Mancha (SESCAM) Health Care Network. EHR: electronic health record.

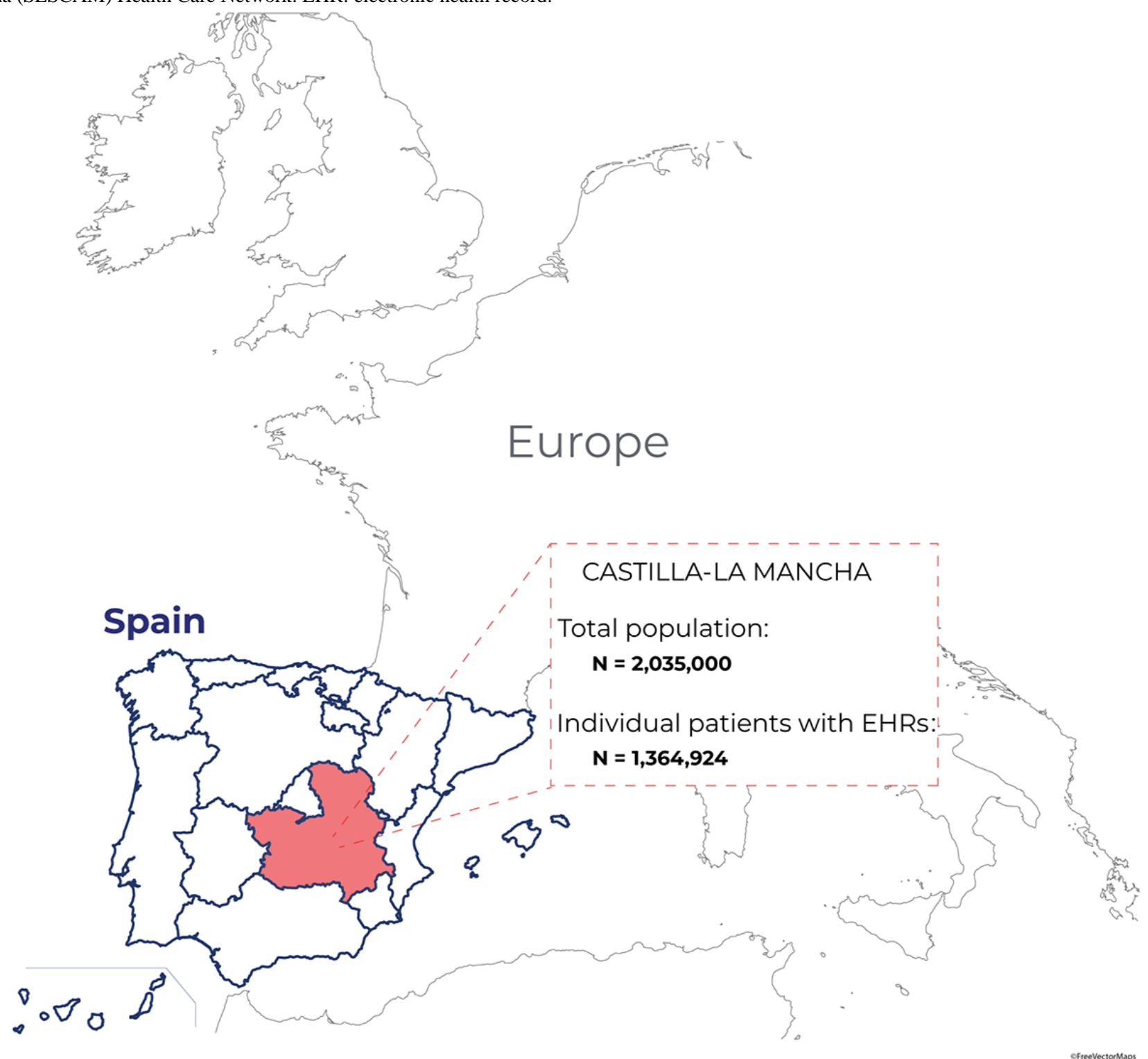

\section{Study Sample}

The study sample included all patients in the source population who were diagnosed with COVID-19. Patients were identified on the basis of clinical diagnosis or microbiological test results. Clinical confirmation of COVID-19 cases was determined by observed symptomatology, imaging (mostly chest X-ray), and laboratory results, as captured in the unstructured, free-text information in the EHRs. Microbiological test result confirmation of COVID-19 cases involved reverse transcriptase-polymerase chain reaction (RT-PCR) or similar available tests. Our decision to consider cases confirmed both clinically and by RT-PCR was justified by the limited availability of routinely administered RT-PCR tests in the region during the study period and supported by recent discussions on the far-from-optimal sensitivity of RT-PCR for COVID-19 (ie, a single negative result from a single specimen cannot exclude the disease in suspected cases) [20,21]. Indeed, recent reports highlight the clinical validity and relatively high sensitivity of symptom- and imaging-based identification of patients with COVID-19, especially in early stages of the disease $[20,22,23]$.

\section{EHRead}

To meet the study objectives, we used EHRead [24], a technology developed by Savana that applies NLP, machine learning, and deep learning to analyze the unstructured free-text information written in millions of deidentified EHRs. This technology enables the extraction of information from all types of EHRs and subsequent normalization of the extracted clinical entities to a unique terminology. This process enables further analysis of a descriptive or predictive nature. Originally based on Systematized Nomenclature of Medicine Clinical Terms (SNOMED CT) terminology, our unique body of terminology comprises more than 400,000 medical concepts, acronyms, and laboratory parameters aggregated over the course of five years of free-text mining, targeting the most common diseases (eg, respiratory diseases, cardiovascular diseases, and diabetes). 
Using a combination of regular expression rules and machine learning models, the terminology entities are detected in the unstructured text and later classified based on sections typically contained in EHRs, hospital services, and other clinical specifications. Importantly, each detected term is described in terms of negative, speculative, or affirmative clinical statements; this is achieved by using deep learning convolutional neural network classification methods that rely on word embeddings and context information (for a similar methodological approach, see [25]). The limitations of case-by-case detection are also overcome with a similar approach to ensure that the detected concepts are used within the appropriate context for the descriptive and predictive analysis.

For particular cases where extra specifications are required (ie, to differentiate COVID-19 cases from other mentions of the term related to fear of the disease or to potential contact), the detection output was manually reviewed in more than 5000 reports to avoid any possible ambiguity associated with free-text reporting. All NLP deep learning models used in this study were validated using the standard training/validation/testing approach; we used a 75/12/13 split ratio in the available annotated data (between 2000 and 3000 records, depending on the model) to ensure efficient generalization on unseen cases. For all developed models, we obtained F scores greater than 0.89 .

\section{Data Analyses}

All categorical variables (eg, comorbidities, symptoms) are shown in frequency tables, whereas continuous variables (eg, age) are described via summary tables that include the mean, $\mathrm{SD}$, median, minimum and maximum, and quartiles of each variable. To test for possible statistically significant differences in the distribution of categorical variables between study groups (ie, male vs female, ICU admission vs no ICU admission), we used Yates-corrected chi-square tests. For continuous variables, mean differences were tested using $t$ tests. Given our general population approach and our unusually large sample size, we were interested in exploring sex-related differences in patients with COVID-19; therefore, most results were stratified by sex [26]. All statistical inferences were performed at the 5\% significance level using 2-sided tests or 2-sided CIs.

\section{Predictive Model}

We developed a decision tree to classify patients with COVID-19 according to their risk of being admitted to the ICU. The two types of patients or classes considered in the model were therefore "admitted to the ICU" and "not admitted to the ICU." The model maps the characteristics of the patients (the variables) to their class in the shape of a tree. From a clinical perspective, this model contemplates all patient variables upon admission; therefore, it is predictive from symptom debut until outcome. The tree is composed of nodes that branch to subsequent child nodes depending on the patient's variables. The tree is built in such a way that each branch separates the two classes as much as possible. This separation is measured as the Shannon entropy, where a node with an entropy of zero indicates that the classification is perfect (either all or none of the patients were admitted to the ICU) and an entropy of one is the worst possible mix $(50 \% / 50 \%)$ [27].

\section{Model Training and Validation}

The model was developed and tested on the available data from hospitalized patients who had or had not been admitted to the ICU; the latter were either discharged from the hospital or died in the course of the disease. This amounted to a total of 900 patients. We validated our algorithm by splitting our COVID-19 sample into a $70 \%$ training set and a $30 \%$ validation set. This means that the model was trained with 630 patients (582 who did not require intensive care vs 48 who did) and validated over the remaining 270 patients. Because the two classes were unbalanced (far fewer patients required ICU admission), we used the standard technique of oversampling the lower class to guarantee a balance of accuracy and recall (ie, the tradeoff between false positives vs false negatives). Further, we sought to replicate the results of this validation in an a posteriori sensitivity analysis, as per recent recommendations for predictive modeling in COVID-19 [28] and TRIPOD (Transparent Reporting of a multivariable prediction model for Individual Prognosis or Diagnosis) guidance [29]. For this second validation, we trained the model with data from the provinces of Ciudad Real and Guadalajara (38\% of the study sample from Castilla-La Mancha), and we used an independent set with combined data set from the other three provinces, namely Toledo, Cuenca, and Albacete, for validation.

Additional details regarding the development and validation of the predictive algorithm are included in the Supplementary Methods in Multimedia Appendix 1. The workflow used for the generation of the predictive algorithm is summarized in Figure S1 in Multimedia Appendix 1.

\section{Results}

From a source general population of 2,035,000 inhabitants, we used NLP and machine learning to analyze the clinical information contained in the EHRs of 1,364,924 anonymous patients (Figure 1). Among these, we identified a total of 10,504 patients diagnosed with COVID-19 (Figure 2). The flowchart of participation in the study up to hospital admission, ICU admission, or discharge is presented in Figure 2. 
Figure 2. Patient flowchart depicting the total number of inhabitants in the source population, the number (\%) of patients with available EHRs analyzed, the number of patients diagnosed with COVID-19, and of those, the number of hospitalizations and ICU admissions. EHR: electronic health record; ICU: intensive care unit.

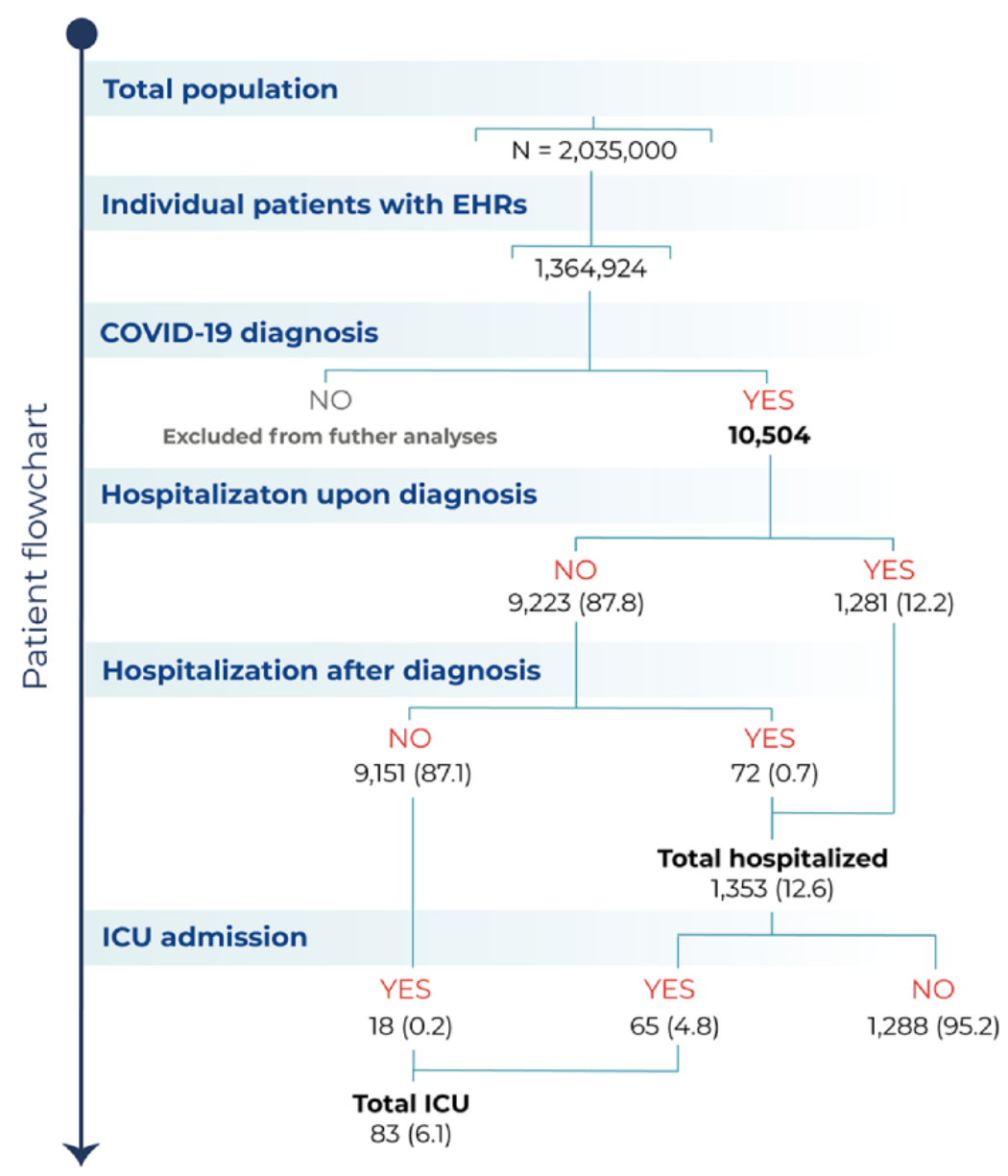

Of the patients with COVID-19, 52.5\% (5519/10,504) were male, with a mean age of 58.2 years (SD 19.7) (Table 1). Most patients with COVID-19 were aged $\geq 50$ years (Figure 3 ). Upon diagnosis, the most common symptoms reported were cough, fever, and dyspnea (Table 1); notably, less than half of patients presented with all three of these symptoms. Further, respiratory crackles, myalgia, and diarrhea were identified in $\geq 5 \%$ of cases, while other respiratory and nonrespiratory signs and symptoms were less common. Sex-dependent differences in sign and symptom frequencies upon diagnosis are shown in Table 1. Of note, we observed subtle increases in the frequency of diarrhea, myalgia, headache, chest pain, and anosmia in female patients with COVID-19, while male patients showed significant increases in fever, dyspnea, respiratory crackles, rhonchus, lymphopenia, and tachypnea (all $P<.05$ ). 
Table 1. Baseline demographics and clinical data of the patients in the study upon diagnosis $(\mathrm{N}=10,504)$.

\begin{tabular}{|c|c|c|c|c|}
\hline Characteristic & Female & Male & Total & $P$ value $^{\mathrm{a}}$ \\
\hline $\operatorname{Sex}^{\mathrm{b}}, \mathrm{n}(\%)$ & 4984 (47.4) & $5519(52.5)$ & $10,504(100)$ & $\mathrm{N} / \mathrm{A}^{\mathrm{c}}$ \\
\hline Age (years) & & & & $<.001$ \\
\hline Mean (SD) & $57.4(20.0)$ & $59.0(19.5)$ & $58.2(19.7)$ & \\
\hline Median (minimum-maximum) & $58.0(0.0-100.0)$ & $60.0(0.0-102.0)$ & $59.0(0.0-102.0)$ & \\
\hline Q1-Q3 & $44.0-73.0$ & $46.0-74.0$ & $45.0-73.0$ & \\
\hline
\end{tabular}

\section{Signs and symptoms, n (\%)}

\begin{tabular}{ll} 
Cough & $2482(49.8)$ \\
Fever & $2120(42.5)$ \\
Dyspnea & $1476(29.6)$ \\
Respiratory crackles & $849(17.0)$ \\
Diarrhea & $556(11.2)$ \\
Myalgia & $467(9.4)$ \\
Headache & $462(9.3)$ \\
Rhonchus & $279(5.6)$ \\
Chest pain & $287(5.8)$ \\
Lymphopenia & $196(3.9)$ \\
Wheezing & $194(3.9)$ \\
Tachypnea & $135(2.7)$ \\
Anosmia & $166(3.3)$ \\
Sore throat & $69(1.4)$ \\
Ageusia & $33(0.7)$ \\
Dysphagia & $19(0.4)$ \\
Neuralgia & $19(0.4)$ \\
Splenomegaly & $8(0.2)$ \\
Hepatomegaly & $2(0.0)$ \\
\hline
\end{tabular}

Comorbidities $^{\mathrm{d}}, \mathbf{n}(\%)$

\section{Cardiovascular disease}

Hypertension

Ischemic stroke

\section{Heart disease}

Ischemic heart disease

Heart failure

Diabetes

Obesity

\section{Renal dysfunction}

$\mathrm{CKD}^{\mathrm{e}}$

Depression

Chronic respiratory disease

Asthma
COPD $^{\mathrm{f}}$
OSA $^{\mathrm{g}}$

2253 (45.2)

1552 (31.1)

$91(1.8)$

1100 (22.1)

$152(3.0)$

243 (4.9)

689 (13.8)

479 (9.6)

$271(5.4)$

$171(3.4)$

484 (9.7)

242 (4.9)

496 (10.0)

$126(2.5)$

69 (1.4)

$2760(50.0)$
$2783(50.4)$
$1818(32.9)$
$1085(19.7)$
$543(9.8)$
$451(8.2)$
$302(5.5)$
$414(7.5)$
$267(4.8)$
$346(6.3)$
$195(3.5)$
$203(3.7)$
$134(2.4)$
$57(1.0)$
$32(0.6)$
$28(0.5)$
$22(0.4)$
$14(0.3)$
$6(0.1)$

$2805(50.8)$

1975 (35.8)

163 (3.0)

1539 (27.9)

475 (8.6)

309 (5.6)

957 (17.3)

457 (8.3)

493 (8.9)

$323(5.9)$

219 (4.0)

$646(11.7)$

263 (4.8)

549 (9.9)

143 (2.6)
5243 (49.9)

4904 (46.7)

3294 (31.4)

1934 (18.4)

1099 (10.5)

919 (8.7)

764 (7.3)

693 (6.6)

554 (5.3)

542 (5.2)

389 (3.7)

338 (3.2)

300 (2.9)

127 (1.2)

65 (0.6)

$47(0.4)$

$41(0.4)$

$22(0.2)$

$8(0.1)$

5058 (48.2)

$<.001$

3527 (33.6)

$<.001$

254 (2.4)

$<.001$

2639 (25.1)

$<.001$

627 (6.0)

$<.001$

552 (5.3)

.11

1646 (15.7)

$<.001$

936 (8.9)

.02

764 (7.3)

$<.001$

494 (4.7)

$<.001$

703 (6.7)

$<.001$

888 (8.5)

$<.001$

759 (7.2)

$<.001$

675 (6.4)

$<.001$

212 (2.0)

$<.001$ 


\begin{tabular}{|c|c|c|c|c|}
\hline Characteristic & Female & Male & Total & $P$ value $^{\mathrm{a}}$ \\
\hline Bronchiectasis & $42(0.8)$ & 87 (1.6) & $129(1.2)$ & $<.001$ \\
\hline Chronic liver disease & $36(0.7)$ & $75(1.4)$ & $111(1.1)$ & .002 \\
\hline Cirrhosis & $16(0.3)$ & $35(0.6)$ & $51(0.5)$ & .03 \\
\hline HIV & $12(0.2)$ & $22(0.4)$ & $34(0.3)$ & .21 \\
\hline
\end{tabular}

${ }^{\mathrm{a}} P$ values from Yates-corrected chi-square test on percentage difference of female vs male COVID-19 patients. All tests were performed individually for each variable (sign, symptom, or comorbidity, where applicable). For numerical values (ie, age), $t$ tests of difference between means were used.

${ }^{\mathrm{b}}$ The sex of one patient was listed as Unknown.

${ }^{\mathrm{c}} \mathrm{N} / \mathrm{A}$ : not applicable.

${ }^{\mathrm{d}}$ List of medical conditions according to Systematized Nomenclature of Medicine Clinical Terms terminology.

${ }^{\mathrm{e}} \mathrm{CKD}$ : chronic kidney disease.

${ }^{\mathrm{f}} \mathrm{COPD}$ : chronic obstructive pulmonary disease.

${ }^{g}$ OSA: obstructive sleep apnea.

Figure 3. Age distribution of incident cases of COVID-19 in females (left) and males (right) in the study population for the period comprised between Jan 1, 2020 and March 29, 2020.

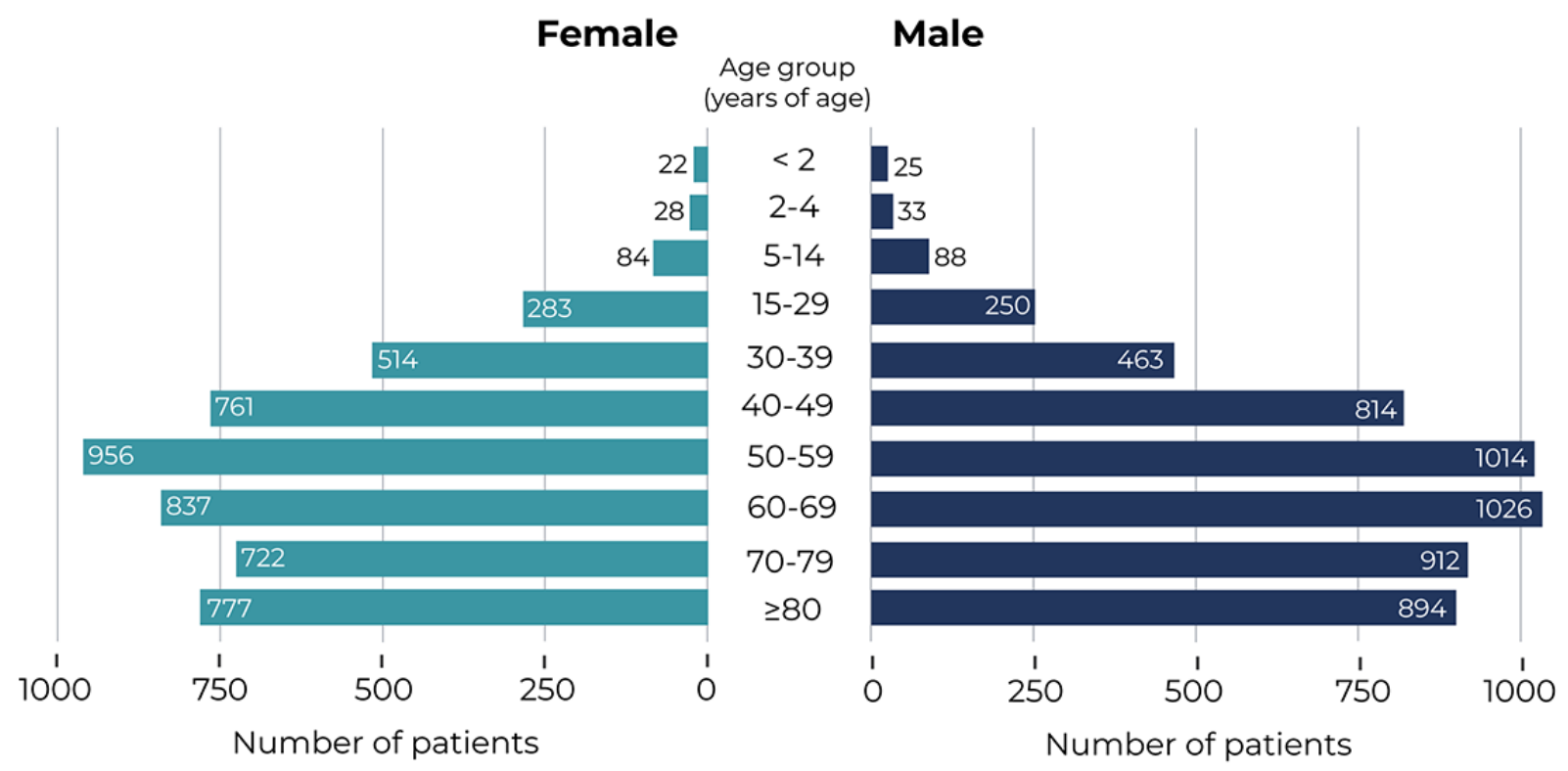

Similarly, the most frequent comorbidities among the 10,504 patients were cardiovascular disease $(n=5058,48.2 \%)$, mainly arterial hypertension $(n=3527,33.6 \%)$; heart disease $(n=2639$, $25.1 \%)$; and diabetes $(\mathrm{n}=1646,15.7 \%)$ (Table 1). Regarding respiratory diseases, COPD was present in 6.4\% (675), asthma in $7.2 \%$ (759), obstructive sleep apnea (OSA) in 2\% (212), and bronchiectasis in $1.2 \%$ (129) of the 10,504 patients. Sex-dependent differences in comorbidities upon diagnosis are also shown in Table 1; except for asthma, the frequency of all comorbidities was significantly higher in male than in female patients with COVID-19 (all $P<.05$ ).

Next, we explored whether the distribution of comorbidities and signs and symptoms captured in the patients' EHRs upon diagnosis differed between patients with COVID-19 who were and were not admitted to the ICU (Table 2). Regarding comorbidities, diabetes, obesity, cardiovascular disease (mainly hypertension), heart disease (mainly ischemic heart disease), and renal dysfunction were more common among patients who were admitted to the ICU (all $P<.01$ ). As for signs and symptoms, cough, fever, dyspnea, respiratory crackles, diarrhea, tachypnea, lymphopenia, and rhonchus were more frequent among ICU patients (all $P<.05$ ). Interestingly, respiratory diseases were not more frequent among patients who were admitted to the ICU (Table 2). 
Table 2. Associations of signs and symptoms and comorbidities with ICU admission upon diagnosis in patients with COVID-19 (N=10,504).

\begin{tabular}{|c|c|c|c|}
\hline Variable & Not admitted to $\mathrm{ICU}^{\mathrm{a}}(\mathrm{n}=10,421), \mathrm{n}(\%)$ & Admitted to ICU (n=83), n (\%) & $P$ value $^{\mathrm{b}}$ \\
\hline \multicolumn{4}{|l|}{ Signs and symptoms } \\
\hline Cough & $5181(49.7)$ & $62(74.7)$ & $<.001$ \\
\hline Fever & $4849(46.5)$ & $55(66.3)$ & $<.001$ \\
\hline Dyspnea & $3246(31.1)$ & $48(57.8)$ & $<.001$ \\
\hline Respiratory crackles & $1904(18.3)$ & $30(36.1)$ & $<.001$ \\
\hline Myalgia & $908(8.7)$ & $11(13.3)$ & .21 \\
\hline Diarrhea & $1084(10.4)$ & $15(18.1)$ & .04 \\
\hline Dysphagia & $47(0.5)$ & $0(0)$ & $>.99$ \\
\hline Wheezing & $383(3.7)$ & $6(7.2)$ & .16 \\
\hline Chest pain & $546(5.2)$ & $8(9.6)$ & .12 \\
\hline Lymphopenia & $524(5)$ & $18(21.7)$ & $<.001$ \\
\hline Headache & $757(7.3)$ & $7(8.4)$ & .84 \\
\hline Rhonchus & $676(6.5)$ & $17(20.5)$ & $<.001$ \\
\hline Hepatomegaly & $8(0.1)$ & $0(0)$ & $>.99$ \\
\hline Anosmia & $297(2.9)$ & $3(3.6)$ & .93 \\
\hline Ageusia & $65(0.6)$ & $0(0)$ & .98 \\
\hline Neuralgia & $41(0.4)$ & $0(0)$ & 1 \\
\hline Sore throat & $126(1.2)$ & $1(1.2)$ & 1 \\
\hline \multicolumn{4}{|l|}{ Comorbidities $^{\mathrm{c}}$} \\
\hline Diabetes & $1613(15.5)$ & $33(39.8)$ & $<.001$ \\
\hline Obesity & $917(8.8)$ & $19(22.9)$ & $<.001$ \\
\hline Chronic respiratory disease & $883(8.5)$ & $5(6)$ & .55 \\
\hline $\operatorname{COPD}^{\mathrm{d}}$ & $673(6.5)$ & $2(2.4)$ & .20 \\
\hline Asthma & $750(7.2)$ & $9(10.8)$ & .29 \\
\hline $\mathrm{OSA}^{\mathrm{e}}$ & $211(2)$ & $1(1.2)$ & .89 \\
\hline Bronchiectasis & $129(1.2)$ & $0(0)$ & .60 \\
\hline Cardiovascular disease & $4998(48)$ & $60(72.3)$ & $<.001$ \\
\hline Hypertension & $3487(33.5)$ & $40(48.2)$ & .007 \\
\hline Ischemic stroke & $253(2.4)$ & $1(1.2)$ & .72 \\
\hline Heart disease & $2604(25)$ & $35(42.2)$ & $<.001$ \\
\hline Ischemic heart disease & $616(5.9)$ & $11(13.3)$ & .01 \\
\hline Heart failure & $548(5.3)$ & $4(4.8)$ & $>.99$ \\
\hline Renal dysfunction & $748(7.2)$ & $16(19.3)$ & $<.001$ \\
\hline $\mathrm{CKD}^{\mathrm{f}}$ & $488(4.7)$ & $6(7.2)$ & .41 \\
\hline Chronic liver disease & $109(1)$ & $2(2.4)$ & .50 \\
\hline Cirrhosis & $51(0.5)$ & $0(0)$ & $>.99$ \\
\hline Depression & $699(6.7)$ & $4(4.8)$ & .64 \\
\hline
\end{tabular}

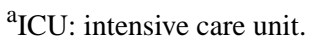


${ }^{\mathrm{b}} P$ values from Yates-corrected chi-square tests of differences between percentages of patients in either outcome group. All tests were performed individually for each variable (sign, symptom, or comorbidity, where applicable).

${ }^{\mathrm{c}}$ List of medical conditions according to Systematized Nomenclature of Medicine Clinical Terms terminology.

${ }^{\mathrm{d}}$ COPD: chronic obstructive pulmonary disease.

eOSA: obstructive sleep apnea.

${ }^{\mathrm{f}} \mathrm{CKD}$ : chronic kidney disease.

Finally, by using a machine-learning, data-driven algorithm, we identified that a combination of three easily available clinical variables, namely age, temperature, and respiratory frequency, was the most parsimonious predictor of ICU admission among patients with COVID-19 (Figure 4). For this model, age and temperature were captured as continuous variables, whereas tachypnea (yes/no) was defined as a respiratory frequency of more than 20 breaths per minute. With accuracy, recall, and area under the curve (AUC) values of 0.68, 0.71, and 0.76, respectively, the presented model reached optimal balance in terms of positive and negative predictive values for ICU admission. On the one hand, patients younger than 56 years, without tachypnea, and with temperature $<39^{\circ} \mathrm{C}$ (entropy $=0$, $\mathrm{n}=145$ ) or $>39^{\circ} \mathrm{C}$ without respiratory crackles (entropy $=0, \mathrm{n}=18$ ) were not admitted to the ICU. On the other hand, patients with COVID-19 aged 40 to 70 years were likely to be admitted in the ICU if they presented with tachypnea and delayed their visit to the ED after being seen in primary care (entropy=0, $n=104$ ). As stated in the Methods section, we performed an additional sensitivity analysis with different data sets to further validate the results of our predictive model. The independent data set of two provinces (Ciudad Real and Guadalajara, including a total of 753,408 individual patients, or $38 \%$ of the entire study sample from Castilla-La Mancha; Figure 1 and Supplemental Table S1 in Multimedia Appendix 1), was used to retrain our algorithm to identify ICU admission at onset; validation was performed in the remaining three provinces. As shown in Supplemental Figure S2 in Multimedia Appendix 1, the new decision tree identified the same relevant clinical variables, that is age, tachypnea, temperature, and respiratory crackles/rhonchus, with similar (but not identical) thresholds in some variables. This additional model achieved accuracy, recall, and AUC values of $0.85,0.57$, and 0.84 , respectively, providing additional proof of validity for our main findings.

Figure 4. Decision tree of relevant clinical variables for the prediction of ICU admission in patients with COVID-19. The combination of three easily available clinical variables, namely age, temperature, and respiratory frequency, was the most parsimonious predictor of ICU admission among COVID-19 patients. The number of patients, probability (p) of ICU admission predicted by the model, and level of entropy (a measure indicating how mixed or pure the classification is, where 0 indicates optimal separation of classes) are indicated in each box. The green pathway indicates that patients with no tachypnea, age $<56$ years, and temperature $<39^{\circ} \mathrm{C}\left(\mathrm{OR}>39^{\circ} \mathrm{C}\right.$ without respiratory crackles) did not require ICU admission. In contrast, the red pathway indicates that patients aged 40-79 years, who presented with tachypnea, and who delayed their visit to the emergency department after being seen in primary care were likely to be admitted in the ICU. For this model, we obtained accuracy, recall, and AUC values of $0.68,0.71$, and 0.76 , respectively (top right panel). AUC: area under the curve; FPR: false positive rate; resp.: respiratory; ROC: receiver operating characteristic; TPR: true positive rate.

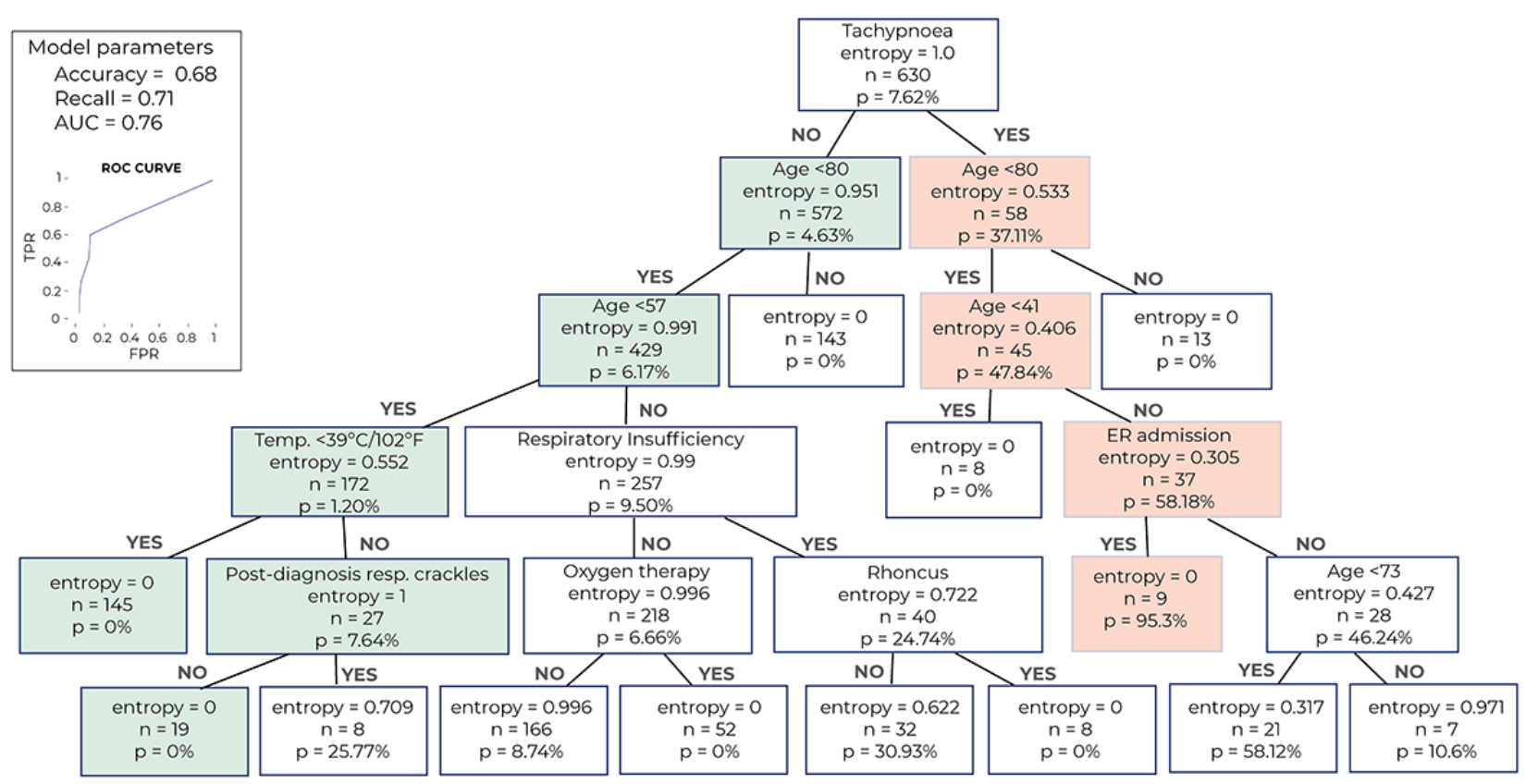

\section{Discussion}

\section{Principal Findings}

By accessing the clinical information of more than 10,000 anonymous patients with COVID-19 (a number that largely surpasses samples included in recent reports about the disease $[30,31]$ ), we were able to describe their demographic and clinical characteristics, their clinical journey, and the statistical relationship between the most common symptoms and comorbidities on admission, and COVID-19 prognosis (ie, ICU admission). There were subtle differences in clinical symptoms 
at onset by sex, while all comorbidities (except asthma) were significantly more frequent in male than female patients with COVID-19. The variables identified in our ICU admission model (ie, age, temperature, and tachypnea) are clinically relevant, as they are readily available and easily observable in everyday practice for patients with COVID-19. Although tachypnea is not an exclusive manifestation of COVID-19 and can be present in patients suffering from other respiratory diseases (ie, pneumonia), our model suggests that this sign (in combination with age and temperature) is a more reliable predictor of ICU use than other common symptoms and signs, such as cough, dyspnea, or respiratory crackles.

The reported sex-dependent differences in the symptomatology of COVID-19 at onset have been further confirmed by our group using similar methods [32] and should be interpreted in light of data suggesting that female teenagers and young adult women are significantly more affected by the disease than their male counterparts [32]. In this regard, our results warrant further investigations aimed at closing the gender gap in the ongoing pandemic [33].

Given that the stability and capacity of ICUs worldwide is threatened by the rapid spread of COVID-19, the identification of individual factors that predict ICU admission may not only improve patient management but also optimize health care resource use and planning. Thus, recent studies using big data and machine learning have explored the prognostic factors of the disease, including ICU transfer, discharge, and mortality [8-11]. In line with our results, respiratory rate has also been identified as an important predictor of ICU transfer in patients with COVID-19 [9].

If further applied to other national and international health care networks, the tools and methodology presented in this study can potentially characterize and predict the prognosis of COVID-19 in a timely and unprecedented manner. As demonstrated in recent studies [34,35], there may be value in the application of AI to the current COVID-19 pandemic, not only to predict outbreaks [36] or read chest computed tomography scans [37] but also to elucidate the clinical onset and natural history of COVID-19 almost in real time. Indeed, classical methods would require months of questionnaire-based data collection and questionnaire validation along with multiple Ethics Board approvals and other practical hurdles; these steps can be avoided with our current approach.

In the race against COVID-19 [38], where the goal is to curb the pandemic, it is imperative to leverage big data and intelligent analytics for the betterment of public health. However, it is of the utmost importance not to neglect privacy and public trust, to apply best practices, and to maintain responsible standards for data collection and data processing on a global scale [39].

\section{Strengths and Limitations}

To our knowledge, this is one of the first attempts to combine NLP and machine learning to access and analyze unstructured, free-text real-world data from EHRs in a large series of patients with COVID-19. Although recent studies have used machine learning to predict ICU admission in patients with COVID-19 [9], our approach takes this methodology one step further by applying NLP to exclusively analyze unstructured information. Indeed, our state-ot-the-art methodology enabled rapid analysis of the unstructured free-text narratives in the EHRs of 1 million patients from the general population of the region of Castilla-La Mancha (Spain).

Our methodology combined modules for sentence segmentation, tokenization, text normalization, acronym disambiguation, negation detection, and a multidimensional ranking scheme; the latter involved linguistic knowledge, statistical evidence, and continuous vector representations of words and documents learned via shallow neural networks. When applied to EHRs, NLP enables both access to longitudinal health records for all patients in the target population and the implementation of exploratory analysis to clarify associations between variables that have remained undetected with traditional research methods. By considering all possible patients with the target disease, the information and analyses used here (ie, real-world data and free-scale statistics) remained unbiased by the research question or the observers. Unlike classical statistical methods (eg, logistic regression), the main advantage associated with the use of machine learning in this context is that it enables the automatic detection of meaningful relationships between variables. For instance, if a given symptom (ie, fever) is only relevant for certain patients (ie, older than 50 years), techniques such as the classification trees used here are suitable to uncover this relationship. In this context, although the total number of patients who required ICU use in the training set was somewhat low (48 patients), the number of variables considered in the model was also very limited. In addition, the inclusion of a validation stage reduces the likelihood of overfitting. Ultimately, the use of classification trees in this study (as opposed to other models, such as artificial neural networks) is especially appropriate in the clinical context because they are easily interpretable.

Regarding the geographical location of our participating hospital sites, it is worth mentioning that with a total of $1,364,924$ patients from the region of Castilla-La Mancha (SESCAM Health Care Network), our sample is representative of the Spanish population. Spain is among the countries that have been most affected by the pandemic in terms of both total cases and mortality rates [40,41]; the Castilla-La Mancha region in particular is the third most affected region in the country, just behind Madrid and Catalonia. For this reason, we anticipate that the clinical conclusions drawn here will be relevant for clinicians worldwide. Of note, the ICU capacity in the region during the study period had not yet been compromised, which protects against possible bias in our training data (all patients requiring intensive care were indeed admitted to the ICU).

The results and conclusions of the present study should be interpreted in light of the following limitations. First, we did not distinguish COVID-19 cases confirmed by laboratory results (ie, RT-PCR) from those exclusively diagnosed through clinical observation (ie, symptomatology, imaging and laboratory results). However, it should be noted that PCR and other rapid laboratory tests for the detection of SARS-CoV-2 were not routinely administered in Spain during the study period. In addition, this decision is supported by recent discussions on the clinical validity and relatively high sensitivity of symptom- and 
imaging-based identification of patients with COVID-19, especially in early stages of the disease [20,22,23]. Second, independent replications by different research groups in larger patient sets are needed to further support the clinical validity of our results.

Finally, future reports from the BigCOVIData study may incorporate laboratory results and treatments and may contextualize the results presented here in a larger clinical picture [28].

\section{Conclusion}

In this study, we found that in the largest series of patients with COVID-19 attended during the first three months of the pandemic in Spain, 6.1\% of all hospitalized patients (83/1353) required ICU admission. We also found that a combination of easily obtained clinical variables, namely age, fever, and tachypnea, predicts whether patients with COVID-19 will require ICU admission.

\section{Acknowledgments}

The members of the Savana COVID-19 Research Group are Ignacio H Medrano, MD; Jorge Tello; Alberto Porras, MD, PhD; Marisa Serrano, PhD; Sara Lumbreras, PhD; Universidad Pontificia Comillas; Carlos Del Rio-Bermudez, PhD; Stephanie Marchesseau, PhD; Ignacio Salcedo; Andrea Martínez; Claudia Maté, MD; Sergio Collazo, MD; Jesús Barea, MD; María Villamayor, MD; Antonio Urda, MD, PhD; Carolina de la Pinta, MD; Imanol Zubizarreta; Yolanda González, PhD; and Sebastian Menke, PhD. We thank all the Savaners for helping accelerate health science with their daily work. This study would have not been possible without every single team member. This study was sponsored by Savana [42]. We also thank the SESCAM Health Care Network in Castilla-La Mancha, Spain, for its participation in the study and for supporting the development of cutting-edge technology in real time.

\section{Conflicts of Interest}

JLI has received consulting or speaking fees from AstraZeneca, Bayer, Boehringer Ingelheim, Chiesi, Glaxo, Grifols, Smith Kline, Menarini, Novartis, Orion, Pfizer, Sandoz, and Teva.

\section{Multimedia Appendix 1}

Supplementary Methods/Supplementary Figures and Tables. [DOC File, 366 KB-Multimedia Appendix 1]

\section{References}

1. Coronavirus (COVID-19). US Centers for Disease Control and Prevention. URL: https://www.cdc.gov/coronavirus/ 2019-ncov/index.html [accessed 2020-04-08]

2. Zhu N, Zhang D, Wang W, Li X, Yang B, Song J, et al. A Novel Coronavirus from Patients with Pneumonia in China, 2019. N Engl J Med 2020 Feb 20;382(8):727-733. [doi: 10.1056/nejmoa2001017]

3. Ferretti L, Wymant C, Kendall M, Zhao L, Nurtay A, Abeler-Dörner L, et al. Quantifying SARS-CoV-2 transmission suggests epidemic control with digital contact tracing. Science 2020 May 08;368(6491):eabb6936 [FREE Full text] [doi: 10.1126/science.abb6936] [Medline: $\underline{\text { 32234805] }}$

4. Qin L, Sun Q, Wang Y, Wu K, Chen M, Shia B, et al. Prediction of Number of Cases of 2019 Novel Coronavirus (COVID-19) Using Social Media Search Index. Int J Environ Res Public Health 2020 Mar 31;17(7):2365 [FREE Full text] [doi: 10.3390/ijerph17072365] [Medline: 32244425]

5. Topol EJ. High-performance medicine: the convergence of human and artificial intelligence. Nat Med 2019 Jan;25(1):44-56. [doi: 10.1038/s41591-018-0300-7] [Medline: 30617339]

6. Divita G, Carter M, Redd A, Zeng Q, Gupta K, Trautner B, et al. Scaling-up NLP Pipelines to Process Large Corpora of Clinical Notes. Methods Inf Med 2018 Jan 23;54(06):548-552. [doi: 10.3414/me14-02-0018]

7. Burgner D, Jamieson SE, Blackwell JM. Genetic susceptibility to infectious diseases: big is beautiful, but will bigger be even better? Lancet Infect Dis 2006 Oct;6(10):653-663. [doi: 10.1016/s1473-3099(06)70601-6]

8. Liu Y, Mao B, Liang S, Yang J, Lu H, Chai Y, et al. Association Between Age and Clinical Characteristics and Outcomes of Coronavirus Disease 2019. SSRN Journal Preprint posted online on March 31, 2020. [doi: 10.2139/ssrn.3556689]

9. Cheng F, Joshi H, Tandon P, Freeman R, Reich DL, Mazumdar M, et al. Using Machine Learning to Predict ICU Transfer in Hospitalized COVID-19 Patients. J Clin Med 2020 Jun 01;9(6):1668 [FREE Full text] [doi: 10.3390/jcm9061668] [Medline: 32492874$]$

10. Nemati M, Ansary J, Nemati N. Machine-Learning Approaches in COVID-19 Survival Analysis and Discharge-Time Likelihood Prediction Using Clinical Data. Patterns (NY) 2020 Aug 14;1(5):100074 [FREE Full text] [doi: 10.1016/j.patter.2020.100074] [Medline: 32835314]

11. Darabi HR, Tsinis D, Zecchini K, Whitcomb WF, Liss A. Forecasting Mortality Risk for Patients Admitted to Intensive Care Units Using Machine Learning. Procedia Computer Science 2018;140:306-313. [doi: 10.1016/j.procs.2018.10.313]

12. Horton R. Offline: COVID-19—what countries must do now. Lancet 2020 Apr;395(10230):1100. [doi: 10.1016/s0140-6736(20)30787-x] 
13. Xu B, Gutierrez B, Mekaru S, Sewalk K, Goodwin L, Loskill A, et al. Epidemiological data from the COVID-19 outbreak, real-time case information. Sci Data 2020 Mar 24;7(1):106 [FREE Full text] [doi: 10.1038/s41597-020-0448-0] [Medline: $\underline{32210236]}$

14. IHME COVID-19 health service utilization forecasting team, Murray CJL. Forecasting COVID-19 impact on hospital bed-days, ICU-days, ventilator-days and deaths by US state in the next 4 months. medRxiv Preprint posted online on March 30, 2020. [doi: 10.1101/2020.03.27.20043752]

15. Sotgiu G, Gerli AG, Centanni S, Miozzo M, Canonica GW, Soriano JB, et al. Advanced forecasting of SARS-CoV-2-related deaths in Italy, Germany, Spain, and New York State. Allergy 2020 Jul;75(7):1813-1815 [FREE Full text] [doi: 10.1111/all.14327] [Medline: 32306406]

16. Izquierdo J, Morena D, González Y, Paredero J, Pérez B, Graziani D, et al. Clinical Management of COPD in a Real-World Setting. A Big Data Analysis. Arch Bronconeumol 2020 Feb 22:in press. [doi: 10.1016/j.arbres.2019.12.025] [Medline: $\underline{32098727]}$

17. Sociedad EDNYCT. Chart Review of Patients With COPD, Using Electronic Medical Records and Artificial Intelligence (BigCOPData). ClinicalTrials.gov. 2019 Dec 20. URL: https://clinicaltrials.gov/ct2/show/NCT04206098 [accessed 2020-04-13]

18. Koo D, Thacker SB. In snow's footsteps: Commentary on shoe-leather and applied epidemiology. Am J Epidemiol 2010 Sep 15;172(6):737-739. [doi: 10.1093/aje/kwq252] [Medline: 20720100]

19. von Elm E, Altman DG, Egger M, Pocock SJ, Gøtzsche PC, Vandenbroucke JP, STROBE Initiative. The Strengthening the Reporting of Observational Studies in Epidemiology (STROBE) statement: guidelines for reporting observational studies. Bull World Health Organ 2007 Nov;85(11):867-872 [FREE Full text] [doi: 10.2471/blt.07.045120] [Medline: 18038077]

20. Long C, Xu H, Shen Q, Zhang X, Fan B, Wang C, et al. Diagnosis of the Coronavirus disease (COVID-19): rRT-PCR or CT? Eur J Radiol 2020 May;126:108961 [FREE Full text] [doi: 10.1016/j.ejrad.2020.108961] [Medline: 32229322]

21. Wang W, Xu Y, Gao R, Lu R, Han K, Wu G, et al. Detection of SARS-CoV-2 in Different Types of Clinical Specimens. JAMA 2020 May 12;323(18):1843-1844 [FREE Full text] [doi: 10.1001/jama.2020.3786] [Medline: 32159775$]$

22. Ai T, Yang Z, Hou H, Zhan C, Chen C, Lv W, et al. Correlation of Chest CT and RT-PCR Testing for Coronavirus Disease 2019 (COVID-19) in China: A Report of 1014 Cases. Radiology 2020 Aug;296(2):E32-E40 [FREE Full text] [doi: 10.1148/radiol.2020200642] [Medline: 32101510]

23. Xu J, Wu R, Huang H, Zheng W, Ren X, Wu N, et al. Computed Tomographic Imaging of 3 Patients With Coronavirus Disease 2019 Pneumonia With Negative Virus Real-time Reverse-Transcription Polymerase Chain Reaction Test. Clin Infect Dis 2020 Jul 28;71(15):850-852 [FREE Full text] [doi: 10.1093/cid/ciaa207] [Medline: 32232429]

24. Hernandez Medrano I, Tello Guijarro J, Belda C, Urena A, Salcedo I, Espinosa-Anke L, et al. Savana: Re-using Electronic Health Records with Artificial Intelligence. IJIMAI 2018;4(7):8. [doi: 10.9781/ijimai.2017.03.001]

25. Yang Z, Dehmer M, Yli-Harja O, Emmert-Streib F. Combining deep learning with token selection for patient phenotyping from electronic health records. Sci Rep 2020 Jan 29;10(1):1432 [FREE Full text] [doi: 10.1038/s41598-020-58178-1] [Medline: $\underline{\text { 31996705] }}$

26. The Lancet. The gendered dimensions of COVID-19. Lancet 2020 Apr;395(10231):1168. [doi: 10.1016/s0140-6736(20)30823-0]

27. Quinlan JR. Induction of decision trees. Mach Learn 1986 Mar;1(1):81-106. [doi: 10.1007/bf00116251]

28. Wynants L, Van Calster B, Collins GS, Riley RD, Heinze G, Schuit E, et al. Prediction models for diagnosis and prognosis of covid-19 infection: systematic review and critical appraisal. BMJ 2020 Apr 07;369:m1328. [doi: 10.1136/bmj.m1328] [Medline: $\underline{\text { 32265220] }}$

29. Moons KG, Altman DG, Reitsma JB, Ioannidis JP, Macaskill P, Steyerberg EW, et al. Transparent Reporting of a multivariable prediction model for Individual Prognosis Or Diagnosis (TRIPOD): Explanation and Elaboration. Ann Intern Med 2015 Jan 06;162(1):W1. [doi: 10.7326/m14-0698]

30. Lescure F, Bouadma L, Nguyen D, Parisey M, Wicky P, Behillil S, et al. Clinical and virological data of the first cases of COVID-19 in Europe: a case series. Lancet Infect Dis 2020 Jun;20(6):697-706. [doi: 10.1016/s1473-3099(20)30200-0]

31. Li Q, Guan X, Wu P, Wang X, Zhou L, Tong Y, et al. Early Transmission Dynamics in Wuhan, China, of Novel Coronavirus-Infected Pneumonia. N Engl J Med 2020 Mar 26;382(13):1199-1207. [doi: 10.1056/nejmoa2001316]

32. Ancochea J, Izquierdo J, Savana COVID-19 Research Group, Soriano J. Evidence of gender bias in the diagnosis and management of COVID-19 patients: A Big Data analysis of Electronic Health Records. medRxiv Preprint posted online on July 26, 2020. [doi: 10.1101/2020.07.20.20157735]

33. Wenham C, Smith J, Morgan R. COVID-19: the gendered impacts of the outbreak. Lancet 2020 Mar;395(10227):846-848. [doi: 10.1016/s0140-6736(20)30526-2]

34. McCall B. COVID-19 and artificial intelligence: protecting health-care workers and curbing the spread. Lancet Digit Health 2020 Apr;2(4):e166-e167. [doi: 10.1016/s2589-7500(20)30054-6]

35. Du RH, Liang LR, Yang CQ, Wang W, Cao TZ, Li M, et al. Predictors of mortality for patients with COVID-19 pneumonia caused by SARS-CoV-2: a prospective cohort study. Eur Respir J 2020 May;55(5):2000524. [doi:

10.1183/13993003.00524-2020] [Medline: 32269088] 
36. Ayyoubzadeh S, Ayyoubzadeh S, Zahedi H, Ahmadi M, Kalhori SRN. Predicting COVID-19 Incidence Through Analysis of Google Trends Data in Iran: Data Mining and Deep Learning Pilot Study. JMIR Public Health Surveill 2020 Apr 14;6(2):e18828 [FREE Full text] [doi: 10.2196/18828] [Medline: $\underline{32234709]}$

37. Li L, Qin L, Xu Z, Yin Y, Wang X, Kong B, et al. Using Artificial Intelligence to Detect COVID-19 and Community-acquired Pneumonia Based on Pulmonary CT: Evaluation of the Diagnostic Accuracy. Radiology 2020 Aug;296(2):E65-E71 [FREE Full text] [doi: 10.1148/radiol.2020200905] [Medline: $\underline{32191588]}$

38. Editorial. The race against COVID-19. Nat Nanotechnol 2020 Apr 17;15(4):239-240. [doi: 10.1038/s41565-020-0680-y] [Medline: 32303704]

39. Ienca M, Vayena E. On the responsible use of digital data to tackle the COVID-19 pandemic. Nat Med 2020 Apr 27;26(4):463-464 [FREE Full text] [doi: 10.1038/s41591-020-0832-5] [Medline: 32284619]

40. Coronavirus disease 2019 (COVID-19) Situation Report 64. World Health Organization. 2020 Mar 24. URL: https://www. who.int/docs/default-source/coronaviruse/situation-reports/20200324-sitrep-64-covid-19.pdf?sfvrsn=723b221e 2 [accessed 2020-03-24]

41. Situación de COVID-19 en España. Ministerio de Sanidad 2020. URL: https://covid19.isciii.es [accessed 2020-04-13]

42. Savana. URL: https://www.savanamed.com/ [accessed 2020-10-23]

\author{
Abbreviations \\ AI: artificial intelligence \\ AUC: area under the curve \\ COPD: chronic obstructive pulmonary disease \\ ED: emergency department \\ EHR: electronic health record \\ ICH: International Council for Harmonisation of Technical Requirements for Pharmaceuticals for Human Use \\ ICU: intensive care unit \\ NLP: natural language processing \\ OSA: obstructive sleep apnea \\ RT-PCR: reverse transcriptase-polymerase chain reaction \\ SESCAM: Servicio de Salud de Castilla-La Mancha \\ SNOMED CT: Systematized Nomenclature of Medicine Clinical Terms \\ STROBE: STrengthening the Reporting of OBservational studies in Epidemiology \\ TRIPOD: Transparent Reporting of a multivariable prediction model for Individual Prognosis or Diagnosis
}

Edited by G Eysenbach; submitted 29.06.20; peer-reviewed by C Fincham, F Palmieri, I Mircheva, J Li; comments to author 21.07.20;
revised version received 28.07.20; accepted 20.10.20; published 28.10.20
Please cite as:
Izquierdo JL, Ancochea J, Savana COVID-19 Research Group, Soriano JB
Clinical Characteristics and Prognostic Factors for Intensive Care Unit Admission of Patients With COVID-19: Retrospective Study
Using Machine Learning and Natural Language Processing
JMed Internet Res 2020;22(10):e21801
URL: $\underline{\text { http://www.jmir.org/2020/10/e21801/ }}$
doi: $10.2196 / 21801$
PMID: $\underline{3090964}$

(CJose Luis Izquierdo, Julio Ancochea, Savana COVID-19 Research Group, Joan B Soriano. Originally published in the Journal of Medical Internet Research (http://www.jmir.org), 28.10.2020. This is an open-access article distributed under the terms of the Creative Commons Attribution License (https://creativecommons.org/licenses/by/4.0/), which permits unrestricted use, distribution, and reproduction in any medium, provided the original work, first published in the Journal of Medical Internet Research, is properly cited. The complete bibliographic information, a link to the original publication on http://www.jmir.org/, as well as this copyright and license information must be included. 